\title{
Diabetes gestacional, hipotiroidismo y concentracion urinaria de yodo en embarazadas. Yodurias en escolares en Paraguay. Exceso de yodo en la sal y riesgo de hiper e hipotiroidismo
}

\author{
Gestational diabetes, hypothyroidism and iodine \\ urinary concentration in pregnant women. \\ Urinary iodine in schoolage children in Paraguay. \\ lodine excess in salt and hyper and hypothyroidism risk.
}

\begin{abstract}
The main indicator of iodine nutritional status of salt for human consumption is the urinary concentration of iodine, which is useful in monitoring universal levels in salt. Paraguay has suffered deficiency in salt with high prevalence of goiter in the population reaching $48.6 \%$. In 2000, results of the Thyroid Mobile project in Latin America showed a goiter prevalence of $17 \%$ but $79.6 \%$ of the consumed iodized salt was adequate in paraguayan households (more than 15 ppm of iodine). The average values of iodine urinary concentration in 4487 scholars was $437 \mu \mathrm{g} / \mathrm{L}, 30 \%$ of the average value was between the ideal of 100 to $199 \mu \mathrm{g} / \mathrm{L}$ and $46.1 \%$ were above $300 \mathrm{mg} / \mathrm{L}$, with risk of excess iodine. Urinary concentration of iodine during pregnancy was measure for the first time in Paraguay in 200 women; the average was $484 \mu \mathrm{g} / \mathrm{L}$ but diabetes and hypothyroidism during pregnancy was observe in 50\% of them. Objective: Determine iodine concentration in urine of the school population in 17 departments of the country and measure the levels of iodine in urine in 200 pregnant women from 15-37 years of age and their levels of glycaemia, and thyroid TSH. Methods: During the years, 2006 and 2007 were evaluated 4487 school randomized, in a probabilistic sample, 3198 in rural areas and 1,289 in urban areas; casual urine samples was collected to determine iodine content. The urinary concentration of iodine in two hundred pregnant women, concentration of iodine in salt, and levels of glycaemia and TSH were also included in the present study. All candidates agreed with the informed consent under the ethics rules. Results: These data showed by comparing the levels of iodine in urine concentration in more than adequate and excessive level but this last was markedly elevated (93.8\%). The median level greater than 300 $\mu \mathrm{g} / \mathrm{L}$ was observed in $100 \%$ of the 4,487 urine samples, while the median urinary iodine levels of $300-500 \mu \mathrm{g} / \mathrm{L}$ was $91.3 \%$ and above 500 was $9.7 \%$. These levels showed the risk of developing thyroid autoimmune diseases. In 200 pregnancy women the average of urine concentration of iodine was $498 \mu \mathrm{cg} / \mathrm{L}$, subclinical and clinical hypothyroidism and gestational diabetes was $50 \%$ of both. Conclusion: To obtain normal median urinary iodine levels is necessary to decrease the amount of iodine in salt. The main recommendation is to insist on adequate monitoring of iodized salt consumption. Also continue surveillance and monitoring in sentinel sites reporting the importance of adequate iodine intake of the population especially in pregnant women that also need to be warm about the diabetes during pregnancy but also a regimen is necessary to keep them within the normal levels of glycemia. The subclinical hypothyroidism has been also studied in the same patients.

Key words: iodine nutrition, schoolchildren, salt concentration of iodine. pregnancy, gestational diabetes.
\end{abstract}

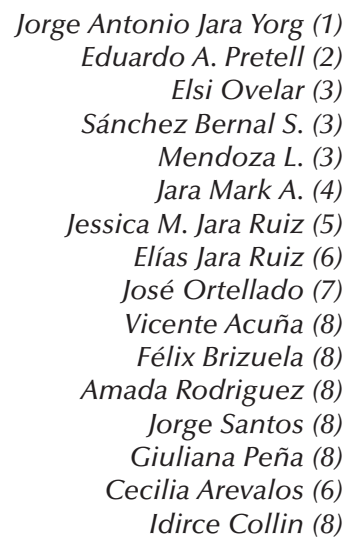

(1) Cátedra de Patología Médica, Universidad Nacional de Asunción, Centro de Diagnóstico y Tratamiento Nuclear (CEDIN), Representante y Coordinador Nacional de lodine Global Network en el Paraguay. Asunción, Paraguay. (2) Coordinador Latinoamericano del lodine Global Network., Universidad Cayetano Heredia, Lima, Perú. (3) MSP y BS, INAN (Instituto Nacional de Alimentación y Nutrición). Asunción, Paraguay (4) University of Buffalo, NY, USA. (5) Universidad Federal de Curitiba, Brasil. (6) Facultad de Ciencias Médicas, Universidad Nacional de Asunción, Paraguay. (7) Programa de Prevención Cardiovascular, MSP y BS, Asunción, Paraguay. (8) Hospital San Pablo, MSP y BS, Asunción, Paraguay.

Dirigir la correspondencia a: Profesor Dr.

Jorge Antonio Jara Yorg Tel:+595-21-297462, Cel.+595981976421 e-mail: jrg.jara@gmail.com

Este trabajo fue recibido el 3 de Marzo de 2015 aceptado con indicaciones el 25 de Agosto de 2015 y aceptado para ser publicado el 28 de Diciembre de 2015. 


\section{INTRODUCCIÓN}

El concepto clásico de considerar sólo a la desnutrición como problema de salud pública en el campo de la nutrición, ha cambiado radicalmente. Es evidente que el hambre y su consecuencia directa, la desnutrición, si bien ha disminuido, aun sigue afectando a millones de seres humanos en todo el mundo, incluyendo a nuestros países de América Latina (1-2). La carencia de yodo, así como la de hierro, además de producir graves trastornos de la salud, son también responsables de problemas socioeconómicos, debido a que producen disminución de la capacidad intelectual y física de las poblaciones afectadas, condicionando el desarrollo humano, económico y social (3-4).

Los Desórdenes por Deficiencia de Yodo (DDI), han sido en el Paraguay, un problema endémico debido a su posición geográfica. Por ser un país mediterráneo muy alejado de las costas marinas, por la geología de su terreno que contiene poco yodo y por no contar con minas de sal se ve obligado a importar el $100 \%$ de la sal, tanto de consumo humano como animal e industrial. El principal indicador del estado nutricional del yodo y del impacto de la yodación de la sal de consumo humano es la concentración urinaria de yodo(56). En la práctica, se mide el yodo en una muestra ocasional de orina, pues se ha demostrado que, salvo en determinadas circunstancias, la medición del cociente yodo/creatinina (Y/C) en una muestra ocasional de orina permite evaluar la excreción urinaria de yodo en 24 horas (7).

Los DDI son varios, y se deben esencialmente a la deficiente producción de hormonas tiroideas. Se caracterizan por disminuir la capacidad física e intelectual de las personas que los padecen (5). El bocio endémico es el trastorno por deficiencia de yodo más frecuente, por lo cual el esfuerzo a nivel nacional en lograr la yodación de la sal de consumo familiar ha sido incesante y sostenido con campañas de yodación que han logrado que las plantas salineras y de expendio pudieran agregar yodo a la sal logrando mejorar esta deficiencia presente por muchos años.

Durante décadas el país ha sufrido déficit de yodo en la sal lo cual determinó una elevada prevalencia de bocio en la población alcanzando 48.6\% de la población escolar. En 1988, el Ministerio de Salud Pública y Bienestar Social (8) lo detectó mediante una encuesta a nivel nacional. En esa oportunidad se realizó palpación de tiroides y niveles de yoduria (8-11). La estrategia principal para la eliminación sostenida de los DDI recomendada ha sido ser la yodación de la sal en forma universal. La ingesta mínima diaria de yodo recomendada para adultos es de $150 \mu \mathrm{g}$ (220 $\mu \mathrm{g}$ para gestantes) (12) La yodación de la sal (con $76 \mu$ g de yodo por gramo) es una medida de salud pública encaminada a paliar este déficit (13).

En el año 2000, los resultados del proyecto Tiroides Móvil en América Latina demostró que $79,6 \%$ de la sal yodada consumida en los hogares paraguayos era adecuada, (más de $15 \mathrm{ppm}$ ) y que la media de los valores de los niveles urinarios fue de $258 \mu \mathrm{g} / \mathrm{L}$, teniendo en cuenta que 30\% de la media se encontraba entre el valor ideal de 100 a $199 \mu \mathrm{g} / \mathrm{L}$ y que $46,1 \%$ estaba por sobre $300 \mu \mathrm{g} / \mathrm{L}$, lo cual implicaba el riesgo de exceso de yodo. La prevalencia de bocio realizada por el método ecográfico demostró una disminución al 17\% (14).

Una consecuencia del exceso de yodo es el hipertiroidismo que sucede con frecuencia en sujetos de edad avanzada con bocios nodulares, pero el hipertiroidismo de Graves y el hipotiroidismo de Hashimoto ocurren también en personas con niveles de yodo en orina por sobre de $300 \mu \mathrm{g} / \mathrm{L}$. El hipertiroidismo inducido por yodo se observa con frecuencia en pacientes afectados por bocio eutiroideo con deficiencia de yodo, cuando repentinamente son expuestos a exceso de yodo. Posiblemente la presencia de la función tiroidea autónoma permite la síntesis y liberación de cantidades excesivas de hormonas tiroideas. En áreas con suficiencia de yodo, el hipertiroidismo inducido por el yodo ha sido reportado en pacientes eutiroideos con previa enfermedad tiroidea, y en pacientes previamente tratados con fármacos antitiroideos por la enfermedad de Graves. Además, el exceso de yodo en pacientes con hipertiroidismo de Graves puede reducir la eficacia de los fármacos antitiroideos, por lo cual el control sostenido y el monitoreo constante es necesario a fin de evitar tanto el déficit como el exceso de los niveles de yodo en la sal (15-17).

Teniendo en cuenta esto, se ha estudiado una población diversa en el área rural y urbana a fin de obtener los valores de yoduria.

Un objetivo de este trabajo fue determinar el estado nutricional del yodo midiendo los niveles urinarios de la población escolar en 17 departamentos del Paraguay, para evaluar el alcance logrado con los programas del ICCIDD y del Ministerio de Salud con la implementación de la yodación de la sal de consumo familiar, las características de la población y los niveles urinarios alcanzados con las sales yodadas de expendio por industrias nacionales de producción. La mediana de la concentración urinaria de yodo en orina es un biomarcador de la ingesta de yodo.

La Organización Mundial de la Salud (OMS) determina que la mediana urinaria de la ingesta de yodo en el rango de 100-199 $\mu \mathrm{g} / \mathrm{L}$ es adecuada y el rango entre $200-299 \mu \mathrm{g} / \mathrm{L}$ es más que adecuada. Se conoce que la tiroglobulina es un promisorio biomarcador tanto de la deficiencia como del exceso de yodo (18).

El hipotiroidismo subclínico en el embarazo es definido como aquel que presenta un nivel de TSH por sobre el rango de referencia y con una concentración de L-Tiroxina dentro de límites normales. Ocurre en 4\% en China, 6,8\% en Bélgica y en el Norte de España en 13,7\%.(19). Es sabido que el hipotiroidismo subclínico produce aborto en $71,4 \%$ de los casos (20), parto prematuro en $7,2 \%$, si hay un tratamiento inadecuado con Levotiroxina. En el hipotiroidismo pleno, el aborto puede ocurrir hasta en $60 \%$ de los casos y el parto prematuro en $20 \%$, sin embargo, las pacientes embarazadas tratadas adecuadamente llegan a término en $100 \%$ para los hipotiroidismos plenos y y en $90,5 \%$ en los hipotiroidismos subclínicos, sin abortos en ninguno de los casos. Las guías de la Asociación Americana y Latinoamericana de Tiroides, consideran en el embarazo niveles normales de TSH bajo 2.50 ( $\mu \mathrm{UI} / \mathrm{I})$ en el primer trimestre, y bajo $3.0(\mu \mathrm{UI} / \mathrm{I})$ en el segundo y tercer trimestres, por los potenciales beneficios, especialmente, en las pacientes con anticuerpos anti TPO positivos. Las dosis de Levotiroxina sódica recomendadas para mantener estos valores de TSH han sido variables y basadas en la práctica de cada profesional. $(21,40)$.

Sabemos que durante el embarazo aumenta la secreción y la resistencia de insulina y disminuye su sensibilidad. En el embarazo normal existe hiperplasia de células Beta (ß) pancreáticas, con aumento de la insulina en el estado inicial y un aumento progresivo de la resistencia a la insulina. En un embarazo con diabetes gestacional hay más resistencia a la insulina y pobre compensación de las células ß. La inhabilidad para incrementar la secreción de insulina en compensación con la insulina resistencia adquirida durante el embarazo resulta en la Diabetes Mellitus Gestacional (22).

El dosaje de los niveles de glucosa o de la prueba a la 
tolerancia a la glucosa es necesario conocer en la mayoría o en todas las mujeres que no se conocen diabéticas. La diabetes mellitus no diagnosticada previamente puede ser ligera y no progresiva (MODY 2) o severa y progresiva (tipo 1, tipo 2 y las formas raras). Una forma de tolerancia a la glucosa alterada es limitada al embarazo o a un estado en la progresión a la diabetes mellitus. En Latinoamérica la incidencia de la DM tipo 1 entre 5 y 10 años alcanza cerca al 60\% (23), por lo tanto, la perspectiva poblacional es identificar a las mujeres jóvenes o relativamente jóvenes con niveles de glucosa en el límite superior durante el embarazo, pues pueden ser portadoras de diabetes, estar en camino a desarrollarla, o no llegar a padecerla nunca. En los EE UU, la diabetes gestacional o la intolerancia a la diabetes afecta al 14\% de las embarazadas, diagnosticadas por primera vez (23).

El peso materno es muy importante en la aparición de la diabetes gestacional y el aumento del mismo está asociado al aumento de la prevalencia de la DMG, como también la raza, siendo mayor la prevalencia entre los asiáticos; y luego los hispanos comparados a los negros y blancos. La diabetes gestacional puede aumentar la hiperglicemia fetal, los ácidos grasos libres e hiperinsulinemia fetal que pueden ocasionar, el aumento de la adiposidad fetal y acidosis, retraso de la maduración pulmonar e hiperglucemia neonatal. También la obesidad infantil, la intolerancia gravídica y la disminución de la función cerebral. Se sabe que el tratamiento de la Diabetes Gestacional incide en la disminución de las complicaciones perinatales severas, de las macrosomia fetal, hipertensión e hiperglucemia neonatal. Para definir el grado de intolerancia a la glucosa se diseñó el HAPO (24).

Considerando que la hiperglicemia puede ser detectada en cualquier momento del embarazo; la American Diabetes Association (ADA) en 2011 determinó que; valores de glicemia en ayunas de 92 a $125 \mathrm{mg} / \mathrm{dL}$ antes de la semana 20, es normal y una glicemia mayor a $126 \mathrm{mg} / \mathrm{dL}$ es considerada como diabetes gestacional lo cual implicaría una pregunta; ¿Sería un nuevo criterio para diagnosticar la prevalencia de DG la identificación de la hiperglicemia antes de las 24 semanas del embarazo? ¿Implicaría un riesgo mayor de complicacio- nes? Sacks y colaboradores encontraron una prevalencia en Canadá el año 2012 del 25\% en 1981 mujeres (25), Trujillo en el Brasil encontró en 4926 mujeres 18\% y el Instituto de Previsión Social en el Paraguay encontró en 1063 una prevalencia de $47 \%$.

En el presente estudio se examinaron a 200 mujeres del Hospital Materno Infantil San Pablo del Ministerio de Salud Pública y Bienestar Social en edades de 15 a 37 años, con promedio de 26 , a quienes se les realizó las pruebas; tolerancia a la glucosa en la primera consulta, el dosaje de TSH, el dosaje de yodo en sal y la yoduria. Estos estudios fueron realizados por primera vez en el país a fin de conocer la prevalencia de diabetes gestacional, hipotiroidismo clínico, subclínico y la relación con el tenor de yodo en la sal y la yoduria (tabla 6), debido a los niveles cercanos al límite máximo de yodo en la sal en los últimos 10 años en el país (tabla 5) de acuerdo a las tablas previamente presentadas.

Todas las embarazadas que presentaron hipotiroidismo subclínico y clínico fueron tratadas con L-Tiroxina Sódica y monitorizadas entre las 4 y 6 semanas después. Se consideró eficaz y adecuado el tratamiento si las embarazadas presentaban un nivel de TSH bajo 2,5 mIU/L en el primer trimestre y bajo $3 \mathrm{mlU} / \mathrm{L}$ en los siguientes trimestres (21).

Objetivos: Determinar los niveles de yodo urinario en escolares de 6-12 años de 17 departamentos del país para evaluar su ingesta de yodo. Determinar los niveles de yodo en sal, yoduria, TSH, glicemia en ayunas y 2 horas post prandial en 200 embarazadas en el Hospital materno infantil San Pablo de Asunción, Paraguay.

Sujetos y Métodos: Se realizó un estudio observacional, descriptivo de corte transversal en escolares de 17 departamentos del país con edades entre 6 -12 años de ambos sexos, obteniendo muestras de orina y preservadas a temperaturas menores de $20^{\circ}\left(-20^{\circ}\right)$ hasta el análisis. Las escuelas fueron seleccionadas de acuerdo a los resultados anteriores, utilizando resultados de evaluaciones previas en el país. Se obtuvo consentimiento informado de los padres.

Sujetos: Desde el año 2006, se obtuvieron muestras de orina de 4.487 niños entre 6 a 12 años de edad (3.198 en

TABLA 1

Yoduria (ppm-partes por millón) - Año 2006.

\begin{tabular}{lccc}
\hline$\%$ ppm & $\begin{array}{c}\text { \% } 19.5 \mathrm{ppm} \\
\text { (déficit) }\end{array}$ & $\begin{array}{c}\%<19.5-20.5 \mathrm{ppm} \\
\text { (normal) }\end{array}$ & $\begin{array}{c}\%>20.5 \mathrm{ppm} \\
(\text { exceso) }\end{array}$ \\
$\mathrm{n}: 44$ & $\mathrm{n}: 0$ & $\mathrm{n}: 194$ & $\mathrm{n}: 3.606$ \\
1.1 & 0.0 & 5.0 & 93.8 \\
\hline ppm: partes por millón. & & & \\
\hline
\end{tabular}

\section{TABLA 2}

Porcentaje de consumo de sal en 2 departamentos del país

(Paraguarí y San Pedro)- Paraguay - Año 2008.

$$
\text { n:409 }
$$

Déficit

Negativo

Exceso

0.4

6.6

93.1 
aéreas rurales y 1.289 en urbanas) de escuelas de 17 departamentos del país, seleccionadas a fin de evaluar el nivel del yodo en el país y conocer si tienen deficiencia, suficiencia, adecuada o excesiva cantidad de yodo en orina conforme a los criterios de la OMS considerando que la fuente de yodo de los niños proviene de la dieta alimentaria local y la sal de consumo familiar.

El año 2015, doscientas embarazadas en el primer trimestre del Hospital San Pablo de Asunción, Paraguay fueron seleccionadas al azar para sus estudios de yoduria, yodo en sal (100 gramos de sal del hogar) glicemia en ayunas y dos horas post prandial y dosaje de TSH. Sus datos antropométricos también fueron incluidos. Consentimiento autorizado fue obtenido de cada una de ellas.

Análisis laboratoriales: La determinación de yodo urinario fue realizada de acuerdo al método de amonio persulfato (Reacción de Sandell-Kothoff.modificado de Pino y Dunn) Los datos fueron obtenidos mediante cálculos estadísticos. La mediana de la concentración de yoduria en niños entre 100 y $299 \mu \mathrm{g} / \mathrm{L}$, define a dicha población como libre de deficiencia de yodo; teniendo en cuenta que, no más del $20 \%$ de las muestras deben tener valores inferiores a $50 \mu \mathrm{g} / \mathrm{L}$. En mujeres durante el embarazo, se considera que la mediana de dicha concentración, entre 150 y $249 \mu \mathrm{g} / \mathrm{L}$, la define de igual manera (26).

\section{RESULTADOS}

En el presente estudio se encontró que 50\% de las 200 embarazadas, del Hospital San Pablo, presentaban diabetes gestacional. Todas las pacientes fueron estudiadas en la primera consulta de su embarazo, y fueron tratadas con un régimen; restricción de hidratos de carbono o insulinoterapia. Aquellas, en cuyo tratamiento no recibieron insulinoterapia, y presentaron, luego del primer trimestre, hiperglicemia persistente aún bajo régimen dietético; bajo consentimiento autorizado, recibieron metformina vía oral y dieta logrando la normalización.

El estudio de las pacientes, abarcó el dosaje de TSH. El resultado obtenido en la primera consulta, durante el primer trimestre, demostró 50\% de hipotiroidismo (40,5\% hipotiroidismo subclínico; 9,5\% hipotiroidismo pleno). Fueron considerados niveles de normalidad, en primer trimestre TSH $<2.5 \mathrm{uUI} / \mathrm{ml}, 2$ do y 3 er trimestre $\mathrm{TSH}<3.0 \mathrm{uUI} / \mathrm{ml}$. Todas las pacientes que presentaron niveles superiores fueron tratadas con Levotiroxina sódica; en ayunas, 75 - 125 mcg/día (tabla

\section{TABLA 3}

Consumo de sal en niños escolares (\%) - Año 2007

17 departamentos del país.(Fuente: Micronutrientes INAN MSP y BS).

$\mathrm{n}: 4.579$
Déficit
Negativo
Normal
Exceso

\section{TABLA 4}

Yoduria en niños escolares - Mediana Urinaria, año 2006.

$\begin{array}{lc}\text { Localidad } & \text { Mediana }(\mu \mathrm{g} / \mathrm{L}) \\ \text { Total } & 437 \\ \text { Concepción } & 453 \\ \text { San Pedro } & 459 \\ \text { Cordillera } & 398 \\ \text { Guaira } & 470 \\ \text { Caaguazú } & 560 \\ \text { Caazapá } & 489 \\ \text { Itapúa } & 441 \\ \text { Misiones } & 314 \\ \text { Paraguarí } & 437 \\ \text { Alto Paraná } & 298 \\ \text { Central } & 466 \\ \text { Ñeembucú } & 375 \\ \text { Amambay } & 312 \\ \text { Canindeyú } & 432 \\ \text { Pte. Hayes } & 360 \\ \text { Alto Paraguay } & 421 \\ \text { Boquerón } & 422\end{array}$

$\mathrm{n}: 4.487$

Número de muestras $\quad \%$

$\begin{array}{cc}4487 & 100 \\ 462 & 10.3 \\ 148 & 3.3 \\ 120 & 2.7 \\ 333 & 7.4 \\ 179 & 9.7 \\ 433 & 9.7 \\ 670 & 14.9 \\ 119 & 2.7 \\ 315 & 7.0 \\ 144 & 3.2 \\ 333 & 7.4 \\ 154 & 3.4 \\ 169 & 3.8 \\ 618 & 13.8 \\ 179 & 4.0 \\ 60 & 1.3 \\ 51 & 1.1\end{array}$


7) por vía oral. Los controles fueron realizados 4 semanas después del inicio del tratamiento, demostrando corrección de sus niveles basales.

\section{DISCUSIÓN}

El yodo es muy necesario para la síntesis y formación de las hormonas tiroideas que procede en su mayor parte del agua y los alimentos como también de productos químicos. La introducción sistemática del consumo de sal yodada como parte de una política activa de prevención de la deficiencia de yodo en los países es el procedimiento más adecuado, sin embargo, es necesario mantener un estricto control y seguimiento de las prácticas con vigilancia sanitaria y el monitoreo de la industria salinera y su expendio al público.

El mayor hallazgo del presente estudio es que el Paraguay pasó de una moderada deficiencia de yodo en el año 1988 a un exceso en su ingesta, demostrada por los elevados niveles de yoduria en escolares de todos los departamentos del país(tablas 1, 2, 3 y 4).

Desde el año 1991, se realizaron campañas de yodación de la sal en el país tratando de lograr la concientización de los responsables en la producción de la sal yodada con niveles óptimos para la población y para la reducción de la prevalencia de bocio. Las plantas salineras que operan en el país administraron las distintas sales de expendio en los comercios con cantidades suficientes de yodo, pero con los años se tornó en excesiva, por lo cual los niveles de yodo deben ser monitorizados.

En el presente estudio, al comparar los niveles de yoduria, se observó que el nivel más que adecuado y el excesivo demuestran que éste ultimo nivel está marcadamente elevado (93.8\%) en los escolares y que la mediana de los niveles mayores a $300 \mu \mathrm{g} / \mathrm{L}$ es $100 \%$ del total de las 4.487 muestras de orina, mientras que en los niveles de $300-500 \mu \mathrm{g} / \mathrm{L}$ es $91,3 \%$ y por sobre 500 fue $9.7 \%$ todo lo cual implica el riesgo de enfermedades tiroideas autoinmunes. En el 2015, al estudiar los niveles de yoduria y considerar el tenor de yodo en la sal en las embarazadas ya en el primer trimestre del embarazo, claramente se observó que en los últimos 10 años permanece el nivel elevado de yoduria, el más elevado en Latinoamérica.

Son necesarias medidas correctivas del nivel de yodo en la sal como el control efectivo de los niveles de yodación de la sal de consumo familiar con monitoreos constantes en diversos lugares del país.

El impacto del exceso de yodo en la sal en las poblaciones vulnerables como son los niños, ancianos y las embarazadas debe ser evaluado. La mayor consecuencia del exceso de yodo es el hipotiroidismo de Hashimoto en personas con niveles de yodo en orina por encima de $300 \mu \mathrm{g} / \mathrm{L}$, pero además el hipertiroidismo inducido por el yodo sucede con frecuencia en los sujetos de edad avanzada con bocios nodulares, sin embargo la enfermedad de Graves- Basedow es la más prevalente en niños, mujeres y ancianos. El hipertiroidismo inducido por yodo se observa con frecuencia en los pacientes afectados por el bocio eutiroideo con deficiencia de yodo, cuando son expuestos a exceso de yodo.

En áreas con suficiencia de yodo, el hipertiroidismo inducido por el yodo ha sido reportado en pacientes eutiroideos con enfermedades anteriores de la glándula tiroides. Pacientes eutiroideos tratados previamente con fármacos antitiroideos por la enfermedad de Graves son propensos a desarrollar hipertiroidismo inducido por yodo. Además, el exceso de yodo en pacientes con hipertiroidismo de Graves puede reducir la eficacia de los fármacos antitiroideos, por lo cual el control sostenido y el monitoreo constante es necesario a fin de evitar

\section{TABLA 5}

Promedio de peso, talla, edad, yoduria, yodo en sal y TSH en mujeres embarazadas-2015.

\begin{tabular}{lccccc}
\hline & & \multicolumn{2}{c}{ n: 200} & & \\
Edad(años) & Peso(Kg) & Talla $(\mathrm{cm})$ & Yoduria $(\mu \mathrm{g} / \mathrm{L})$ & Yodo en sal $(\mu \mathrm{g} / \mathrm{L})$ & $\mathrm{TSH}(\mu \mathrm{UI} / \mathrm{L})$ \\
26 & 75.22 & 159.01 & 484 & 39.7 & 2.89 \\
\hline
\end{tabular}

TABLA 6

Concentración urinaria de yodo en mujeres embarazadas y niños en edad escolar.

\begin{tabular}{|c|c|c|c|c|}
\hline País & Región/ciudad & Año & $M E(\mu \mathrm{g} / \mathrm{L})$ & Escolares $(\mu \mathrm{g} / \mathrm{L})$ \\
\hline Argentina & NOA & 2012 & 119 & 116 \\
\hline Argentina & Buenos Aires & 2005 & 137 & 143 \\
\hline Brasil & Sao Paulo & 2009 & 138 & \\
\hline Guatemala & & 2011 & 125 & 139 \\
\hline México & HIdalgo & 2002 & 116 & 123 \\
\hline México & Querétaro & 2014 & 146 & \\
\hline Perú & Ayacucho & 1998 & 115 & 180 \\
\hline Uruguay & & 1998 & 70 & 119 \\
\hline Paraguay & & 2006 & & 437 \\
\hline Paraguay & Asunción & 2015 & 484 & \\
\hline
\end{tabular}

Datos en la revista Eliminación Sostenible de los Desordenes por Deficiencia de Yodo en Latinoamérica.

Reporte de Talleres Sub Regionales para evaluar la situación de los países de Latinoamérica.ME (mujeres embarazadas). 
tanto el déficit como el exceso de los niveles de yodo en la sal (15-22).

Como conclusión, para mantener los niveles normales de yoduria se debe disminuir el nivel de yodación de la sal. Los resultados de los niveles de la mediana urinaria mostraron que la mediana nacional fue $437 \mu \mathrm{g} / \mathrm{L}$, con una mediana en $17 \mathrm{de}-$ partamentos del 93,8\% del país por encima de $300 \mu \mathrm{g} / \mathrm{L}$, por lo cual la principal recomendación es insistir en el monitoreo de la adecuada yodación de las sales de consumo familiar (2328). Además se debe continuar con la vigilancia y el monitoreo constante en sitios centinelas divulgando la importancia de la adecuada ingesta de yodo a la población (29-33).

La detección del hipotiroidismo subclínico en 50\% de las embarazadas demuestra el elevado número de pacientes que padecerían esta patología en todo el país considerando la sub muestra considerada en el presente estudio. Esto implicaría la necesidad del control de rutina en todas las embarazadas en la primera consulta como también el control de la glicemia en ayunas y post prandial.

Como en el centro hospitalario de mayor afluencia de pacientes en el país(Instituto de Previsión Social) se observó diabetes gestacional en el $47 \%$ de las embarazadas, sin embargo, 50\% de las gestantes en el Hospital San Pablo presentaron niveles elevados de glicemia, por lo que en el resto del país podría presentarse un elevado número de pacientes con diabetes gestacional y asociadas al hipotiroidismo subclínico y clínico debido al elevado tenor de yodo en la sal de consumo diario.

\section{RESUMEN}

El principal indicador del impacto de la yodación de la sal de consumo humano es la concentración urinaria de yodo la cual es útil en el monitoreo de la sal. En la encuesta del año 1988 realizada en el Paraguay, se alcanzó una prevalencia de bocio de $48,6 \%$ en la población escolar con un déficit de yodo en la sal, pero el año 2000 en el estudio del proyecto de Tiroides Móvil, se redujo por el método ecográfico a $17 \%$. Ese mismo año la mediana de los niveles urinarios en niños escolares de 6-12 años fue $258 \mu \mathrm{g} / \mathrm{L}$, considerando que $30 \%$ se encontraba entre el valor ideal de 100 a $199 \mu \mathrm{g} / \mathrm{L}$ y $46,1 \%$ sobre $300 \mu \mathrm{g} / \mathrm{L}$, implicando un riesgo de exceso de yodo en dicha población con las posibles consecuencias de aparición tanto de hipo como de hipertiroidismo. El 93\% presentó exceso de yodo en la sal y la mediana urinaria fue $437 \mathrm{ug} /$ $\mathrm{mL}$. Un grupo de embarazadas han sido estudiadas en el Hospital San Pablo por primera vez en el Paraguay para la determinación de la yoduria, yodo en sal, diabetes gestacional, hipotiroidismo en el embarazo y sus valores antropométricos resultando un hipotiroidismo subclínico del 50\% y diabetes gestacional del 50\%. Objetivo: Determinar las yodurias en la población escolar de 6-12 años pre púber de ambos sexos en 17 departamentos del país y en una submuestra en embarazadas y también niveles de glicemia en el embarazo en un hospital de Asunción. Sujetos y Métodos: En el periodo del 2006-2007 fueron evaluados 4.487 escolares randomizados, en una muestra probabilística, 3.198 en el área rural y 1.289 en el área urbana con muestras en orina casual. El año 2015, doscientas embarazadas fueron estudiadas con muestras de glicemia en ayunas y 2 horas post prandial, dosaje de TSH, yodo en sal de 100 gramos de la sal de consumo en sus hogares y de orina para ver la concentración de yodo en sal y urinaria de yodo. Todos los candidatos fueron estudiados bajo consentimiento autorizado y de acuerdo a normas éticas. Resultados: Al comparar los niveles de yodo en orina en el nivel más que adecuado y el excesivo de la yoduria se vió que éste último nivel estaba marcadamente elevado (93.8\%), que la mediana de los niveles mayores a $300 \mathrm{ug} / \mathrm{L}$ era del $100 \%$ del total de las 4.487 muestras de orina, mientras que la mediana de los niveles de yoduria entre 300-500 $\mu \mathrm{g} / \mathrm{L}$ fue de $91,3 \%$ y por encima de 500 fue $9.7 \%$ lo cual implicó el riesgo de desarrollar enfermedades tiroideas autoinmunes. Las embarazadas presentaron una mediana urinaria de yodo 484 $\mu \mathrm{g} / \mathrm{L}$, diabetes gestacional e hipotiroidismo subclínico en $50 \%$. Conclusión: Para normalizar los niveles de yoduria, es necesario disminuir la cantidad de yodo en la sal. La recomendación es insistir en el monitoreo de la adecuada yodación de las sales de consumo familiar y continuar con la vigilancia y el monitoreo constante en sitios centinelas divulgando la importancia de la adecuada ingesta de yodo a la población. Un régimen

\section{TABLA 7}

Niveles de TSH y dosis de L-tiroxina sódica en 200 mujeres embarazadas - 2015.

\begin{tabular}{lcc}
\hline TSH $\mu \mathrm{UI} / \mathrm{L}$ & Basal $(\mu \mathrm{UI} / \mathrm{L})$ & Dosis de L-T4 $(\mu \mathrm{UI} / \mathrm{L})$ \\
Grupo 1 & $2,5-10(81)(40,5 \%)$ & 75 \\
Grupo 2 & $>10(19)(9,5 \%)$ & 125 \\
Normal & $<2.5(100)(50 \%)$ & \\
\hline
\end{tabular}

\section{TABLA 8}

Promedio de la glicemia (mg/dl) en embarazadas año 2015. $(n=200)$

Ayunas

82

Normal

Diabetes gestacional
2 horas post prandial

118

146

$\mathrm{VN}=92-125(\mathrm{mg} / \mathrm{dl})$ 
dietético a las embarazadas es necesario implementar en el primer trimestre del embarazo.

Palabras clave: Nutrición de yodo, volumen tiroideo, función tiroidea. glicemia en el embarazo, diabetes gestacional, hipotiroidismo en el embarazo.

\section{BIBLIOGRAFÍA}

1. Zimmerman MB. Symposium on 'Geographical and geological influences on nutrition lodine deficiency in industrialised countries. Proc Nutr. Soc. 2010; 69:133-43.

2. Hetzel BS. The concept of iodine deficiency disorders (IDD) and their eradication. In: Dunn JT, Pretell EA, Daza $C H$, Viteri FE (eds) Towards Eradication of Endemic Goiter, Cretinism, and lodine Deficiency. Pan American Health Organization Sc Pub 502, Washington D.C. 1986 pp. 109-14.

3. Nyenwe EA, Dagogo-Jack S. Iodine Deficiency Disorders in the lodine-Replete Environment. Am J Med Sci. 2009; 337(1):37-40.

4. World Health Organization-WHO. International Council for Control of lodine Deficiency Disorders. Assessment of iodine deficiency disorders and monitoring their elimination. A guide programmed managers, 2nd edition. Geneva, Switzerland, WHO, Department of Nutrition for Health and Development; 2001. (WHO/NHD/01.1).

5. de Benoist $B$, McLean $E$, Andersson M. Iodine deficiency in 2007: Global progress since 2003. Food Nutr Bull 2008;29:195-202.

6. Pretell EA, Delange F, Hostalek U, Corigliano S, Barreda $L$, Higa AM. Iodine nutrition improves in Latin America. Thyroid 2004;14:590-9.

7. Rueda $R$, Pardo F. Prevención del bocio endémico en Colombia. Boletín de la oficina Sanitaria Panamericana. Diciembre de 1966:495-503.

8. Millón Ramírez MC. Prevalencia de bocio endémico y otros trastornos relacionados de la deficiencia de yodo en la dieta en la comarca de la Axarquía (Málaga). Ed. Universidad de Málaga; colección Tesis Doctorales. ISBN978-84-7496879-8.

9. Patiño JF. Revisión histórica sobre el bocio en Suramérica y la Nueva Granada. Med (Bogotá) 2001; 23(2): 135-50.

10. Carrillo JC. Temas de Pediatría, Edición Especial, Evaluación de Desórdenes por Deficiencia de Yodo (DDI) en dos poblaciones de Casanare Colombia. Nestlé. Servicio de información científica. 1984-1986.

11. Vigilancia Epidemiológica de los Desórdenes por Deficiencia de Yodo. Colombia, 1.997 Ministerio de Salud/INS/UNICEF/ICBF/INVIMA/OPS/OMS. 1998.

12. Control de los Desórdenes por deficiencia de Yodo en Colombia. Evaluación Externa. Ministerio de Salud/OPS/ OMS. 1998 p 3-8.

13. Vigilancia de concentraciones de yodo en sal, Colombia, 1997-1998. SIVIGILA No 21/Mayo 23 al 29 de 1999; pp:1-11.

14. Estudio Centinela de la Estrategia de Niños Veedores Científicos para Yoduria y Contenido de Yodo en Sal. Acosta J, Alvarez V H, Grupo de salud Pública, Ministerio de Salud. Ortiz J, Nava G, Laboratorio de Salud Ambiental, Instituto Nacional de Salud. Nuñez, S. ICBF. Instituto Nacional de Salud, INVIMA). 1999.

15. Vigilancia de concentraciones de yodo en sal, Colombia. Estudios centinela de yodo y fluor - fase I - 2001; semana epidemiológica no. 49; diciembre 2 al 8 de 2001.

16. Ministerio de Salud, Instituto Nacional de Salud, Instituto Colombiano de Bienestar Familiar, Sociedad Colombiana de Endocrinología, UNICEF-OPS/ OMS, Colciencias. Prevalencia de los Desórdenes por Deficiencia de Yodo e Ingestión Promedio de Sal. ISBN Colombia, 1994-1998. Santa Fe de Bogotá, D.C., primera edición; noviembre de 2001. 958-13-0129-1.

17. Gallego $M L$, Loango $N$, Londoño $A L$, Landázuri P. Niveles de excreción urinaria de yodo en escolares del Quindío, 2006-2007. Rev. Salud Pública 2009;11(6):952-960.

18. WHO/UNICEF/ICCIDD. Assessment of iodine deficiency disorders and monitoring their elimination: a guide for programed managers [updated 1st. September 2008]. 3rd edh. Geneva, Switzerland: World Health Organization, 2007.

19. John Lazarus Rosalind S. Braum, Chantal Daumerie, Alicja Hubalewska-Dydejczyk, Roberto Negro, Bijay Vaidya,2014 European Thyroid Association Guideline for the management of subclinical hypothyroidism in pregnancy and in children, June; 3(2): 76-94. Doi: 10.1159/000362597.

20. Abalovich M. Overt and subclinical hypothyroidism complication pregnancy, Thyroid 2002, 12(1):63-8.

21. Busko M. Optimal Levothyroxine doses for hypothyroidism in pregnancy. Medscape.Dec.2013, art.817459

22. Bergman RN, Phillips LS, Cobelli C., Physiologic evaluation of factors controlling glucose tolerance in man: measurement of insulin sensitivity and beta-cell glucose sensitivity from the response to intravenous glucose. J Clin Invest. 1981; 68(6):1456-67.

23. Kim C, Newton KM, Knopp RH. Gestational diabetes and the incidence of type 2 diabetes: a systematic review, Diabetes Care. 2002 ; 25(10):1862-8

24. Coustan DR1, Lowe LP, Metzger BE, Dyer AR; International Association of Diabetes and Pregnancy Study Groups. The Hyperglycemia and Adverse Pregnancy Outcome (HAPO) study: paving the way for new diagnostic criteria for gestational diabetes mellitus. Am J Obstetric Gynecol. 2010; 202(6):654.e1-6. doi: 10.1016/j. ajog.2010.04.006.

25. David A. Sacks, MD,David R. Hadden, MD Michael Maresh, MD Chaicharn Deerochanawong, MD, Alan $R$. Dyer, PHD, Boyd E. Metzger, MD, Lynn P. Lowe, PHD, Donald R. Coustan, MD, Moshe Hod, MD, Jeremy J.N. Oats, MD, Bengt Persson, MD, PHD, Elisabeth R. Trimble, $M D$, For the HAPO STUDY COOPERATIVE RESEARCH GROUP, Frequency of Gestational Diabetes Mellitus at Collaborating Centers Based on IADPSG Consensus Panel-Recommended Criteria Diabetes Care, Volume 35, March 2012.

26. Zimmerman $M B$, Jooste $P L$, Pandav CS. Iodine deficiency disorders. Lancet 2008; 372:1251-62.

27. Laurberg P, Jorgensen T, Perrild H. The Danish investigation on iodine intake and thyroid disease, DanThyr: status and perspectives. Eur J Endocrinol 2006; 155:219-28.

28. Zimmerman $M B$. The role of iodine in human growth and development. Semin Cell Dev Biol. $2011 ;(6): 645-52$.

29. García Asuero A. Revisión. Los Halógenos, ¿materia mineral farmacéutica? An. R. Acad. Nac. Far 2008; 74(1).

30. Johnson CC, Fordyce FM, Stewart AG. (2003). Environmental controls in lodine Deficiency Disorders Project Summary Report. British Geological survey Commissioned Report CR/03/058N.BGS, Keyworth,Nottingham, UK. http://www.lenntech.es/periodica/elementos/i. htm\#ixzz1VFfeMCYx.

31. Follis RH. Distribución Geográfica de la Deficiencia de Yodo en América Latina.

32. Wolff J. Transport of iodide and other anions in the thyroid 
gland. Physiol. Rev. 1964; 44:45-90.

33. Bizhanova A, Kopp P. Minireview: the sodium-iodide symporter NIS and pendrin in iodide homeostasis of the thyroid. Endocrinology 2009; 150:1084-1090.

34. Bol Oficina Sanitaria Panam Salud 1966: p 28-38.

35. Johnson CC. 2003. The geochemistry of iodine and its application to environmental strategies for reducing the risks from iodine deficiency disorders. British Geologi Surv, CR/03/057N.

36. Skeaff SA. Iodine deficiency in Pregnancy: Effect Neurodevelop Child. Nutrients. 2011; 3(2):265-73.

37. Leung AM, LaMar A, He X, Braverman LE, Pearce EN. lodine Status and Thyroid Function of Boston- Area
Vegetarians and Vegans. J Clin Endocrinol Metab 2011; 96(8):E1303-E130.

38. Zimmermann MB. lodine Deficiency. Endocr. Rev. 2009; 30(4):376-408.

39. Lyn Patrick ND. Iodine: Deficiency and Therapeutic Considerations. Altern Med Rev 2008;13(2):116-27.

40. Alex Stagnaro-Green, Marcos Abalovich, Erik Alexander, Fereidoun Azizi, Jorge Mestman,Roberto Negro,Angelita Nixon, Elizabeth N. Pearce, Offie P. Soldin, Scott Sullivan, and Wilmar Wiersinga, Guidelines of the American Thyroid Association for the Diagnosis and Management of Thyroid Disease During Pregnancy and Postpartum, THYROID Volume 21, Number 10, 2011. 